\title{
Norois
}

Environnement, aménagement, société

223 | 2012

Villes petites et moyennes

\section{La petite ville française au défi de l'enfermement résidentiel}

The small french town with the challenge of residential enclosure

\section{François Madoré}

\section{OpenEdition}

1 Journals

\section{Édition électronique}

URL : https://journals.openedition.org/norois/4181

DOI : $10.4000 /$ norois. 4181

ISBN : 978-2-7535-2043-1

ISSN : $1760-8546$

\section{Éditeur}

Presses universitaires de Rennes

\section{Édition imprimée}

Date de publication : 30 mars 2012

Pagination : 43-62

ISBN : 978-2-7535-2041-7

ISSN : 0029-182X

\section{Référence électronique}

François Madoré, "La petite ville française au défi de l'enfermement résidentiel », Norois [En ligne], 223 | 2012, mis en ligne le 28 février 2014, consulté le 13 janvier 2022. URL : http:// journals.openedition.org/norois/4181; DOI : https://doi.org/10.4000/norois.4181

\section{(c) Tous droits réservés}




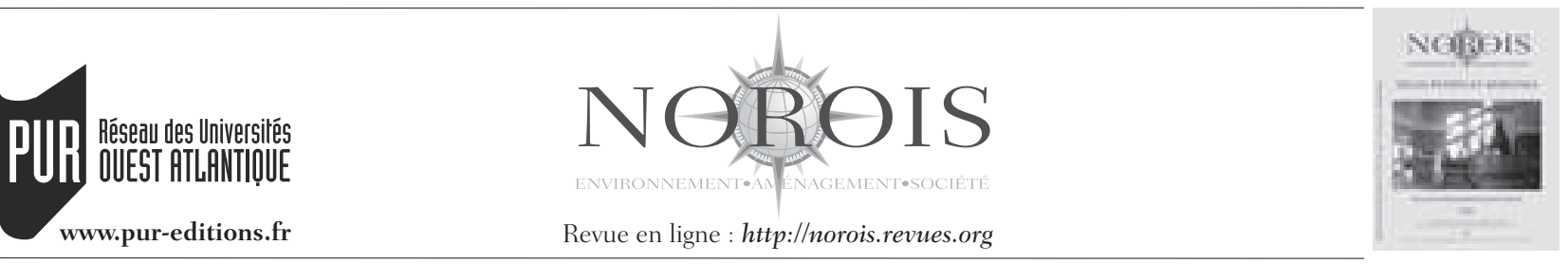

\title{
La petite ville française au défi de l'enfermement résidentiel
}

\author{
The Small French Town with the Challenge of Residential Enclosure
}

\author{
François MADORÉ
}

UMR 6590 ESO-Nantes, Université de Nantes, Igarun, Chemin la Censive du Tertre, BP 81227 - 44312 Nantes

Cedex 3, France (francois.madore@univ-nantes.fr)

Résumé : Les enclaves résidentielles fermées se développent en France, dans les petites villes comme dans les plus grandes. Deux sources sont mobilisées pour étudier ce phénomène. La première est basée sur une identification (sites Web des promoteurs) des programmes immobiliers en cours de commercialisation qui peuvent être considérés comme fermés avec contrôle des accès. La deuxième est fondée sur l'analyse de la diversité typologique des enclaves résidentielles fermées dans deux petites villes de l'ouest Atlantique français. Biscarrosse, dans les Landes, connaît un accroissement démographique très élevé depuis 1999 et une part significative de la construction neuve a eu pour cadre des complexes fermés. À Saint-Martin-de-Ré, en Charente-Maritime, c'est un processus de densification sous la forme d'une urbanisation de clos entourés d'un mur et avec des restrictions d'accès variables qui est observé.

\begin{abstract}
The gated communities grow in France, in small towns and in larger ones. Two methods are mobilized to study the phenomenon. The first inventory tool is based on the identification - in promoters'web-sites - of the real estate programs currently on the market which can be considered as gated and (associated) with control of the access. The second is based on analysis of typological diversity of gated communities in two small towns in the western Atlantic French. Biscarrosse, in the Landes, is experiencing very high population growth since 1999 and a significant share of new construction was closed for part of the complex. In Saint-Martin-de-Ré, Charente-Maritime, is a process of densification in the form of a closed urbanization surrounded by a wall with access restrictions variables is observed.
\end{abstract}

Mots clés : enclaves résidentielles fermées, petites villes, France, Biscarrosse, Saint-Martin-de-Ré

Keywords: gated communities, small town, France, Biscarrosse, Saint-Martin-de-Ré

Les enclaves résidentielles fermées caractérisent de plus en plus le paysage de nos villes, en France comme à l'étranger (Glasze et al., 2002, 2005; Billard et al., 2005; Capron, 2006; Le Goix, Webster, 2008; Paquot, 2009; Billard et al., 2011). L'organisation tous les deux ans d'une conférence internationale centrée sur ce phénomène témoigne de l'intérêt que suscitent, au sein de la communauté des chercheurs entre autres, ces nouveaux territoires de l'habiter construits sur le registre de la fermeture. Toutefois, l'une des difficultés majeures pour nommer ce phénomène en France est précisément l'absence d'appellation générique et acceptée par tous pour définir cette forme d'habitat, contrairement à ce que nous observons dans d'autres contextes géographiques, comme par exemple aux États-Unis avec 
les gated communities, au Brésil avec les condomínios fechados ou encore dans l'Amérique latine hispanique avec les conjuntos cerrados. En fait, dans l'hexagone, la dénomination la plus fréquente est « ensemble, enclave ou complexe résidentiel fermé et/ou sécurisé ».

Bien évidemment, d'aucuns objecteront que ces processus de fermeture dans l'habitat ne sont pas nouveaux, ce qui est exact. Ils existent en effet depuis le XIX ${ }^{\mathrm{e}}$ siècle au moins (Capron, 2006); (Callen et Le Groix, 2007), mais ils ne concernaient alors que quelques domaines d'habitat bourgeois correspondant à la figure du « ghetto doré ». Celle-ci se déploie d'ailleurs en partie seulement sur le registre de la fermeture, car des modes de régulation privée puissants et coercitifs, fondés entre autres sur un strict respect du cahier des charges, régissent les grands lotissements chics de la bourgeoisie en banlieue parisienne (Pinçon-Charlot et Pinçon, 2007). La nouveauté vient donc de l'ouverture du marché de la fermeture résidentielle à la vaste classe moyenne (Donzelot, 1999; Jaillet, 1999). Parallèlement, ces enclaves ont tendance à se diffuser géographiquement et à ne plus être confinées aux seuls territoires d'élection des ménages fortunés, principalement situés dans l'hexagone en région parisienne et sur la Côte d'Azur. Elles ont même tendance à proliférer sur tout le territoire français et pas seulement dans des contextes métropolitains. Les petites villes ne restent donc pas à l'écart de la diffusion de ce modèle.

Cet essor des enclaves résidentielles fermées interroge la façon de vivre en ville et celle de se penser ou de se représenter en société, car il traduit fondamentalement une série de transformations profondes des modes de vie, des relations sociales et des rapports à l'espace. Les forces qui sous-tendent ce phénomène sont par ailleurs très variables. Elles peuvent osciller, selon les contextes géographiques observés à travers le monde, entre recherche de sécurité (Caldeira, 1996, 2001), de tranquillité (Charmes, 2005), d'entre soi (Low, 2003, 2005 ; Chevalier et Carballo, 2004), de nature et de qualité de vie sur fond de rejet des maux de la ville (Giroir, 2002; Raposo, 2003; Lacarrieu et Thuillier, 2004), de distinction et de préservation de la valeur du capital immobilier (Le Goix, 2002) ou encore d'une volonté de construire une économie de club (Webster, 2002). Autant de motivations variées qui, au demeurant, ne sont guère spécifiques aux résidants d'ensembles résidentiels fermés ou sécurisés, comme l'illustrent la plupart des travaux qui interrogent les fondements du phénomène (Capron, 2004).

La finalité de cet article est d'observer la diffusion et surtout la diversité typologique des enclaves résidentielles fermées à l'échelle des petites villes en France. L'approche monographique sera donc privilégiée, afin de décrire finement ce phénomène dans deux petites villes de l'ouest Atlantique, Biscarrosse dans les Landes et Saint-Martin-de-Ré en Charente-Maritime. Notre contribution s'inscrit donc dans la filiation des travaux développés à l'échelle de plusieurs grandes agglomérations françaises, qu'il s'agisse de Lyon (Charmes, 2005), de Marseille (Dorier-Apprill, 2008), de Paris (Charmes, 2005 ; Loudier-Malgouyres, 2007, 2010; Callen et Le Goix, 2007) ou encore de Toulouse (Golovtchenko et Souchet, 2005; Sabatier, 2005). Nous porterons le regard sur un échelon urbain peu étudié, la petite ville, où l'observation de la diversité typologique des complexes résidentiels fermés et des modalités de gestion du contrôle des accès nous aidera à mieux comprendre certains des ressorts du phénomène d'enclosure résidentielle.

\section{LE PLEIN ESSOR DES ENCLAVES RÉSIDENTIELLES FERMÉES}

\section{Les grandes villes n'ont pas l'exclusivité des enclaves résidentielles fermées}

L'hypothèse d'une large diffusion géographique des enclaves résidentielles fermées en France, bien au-delà des seuls territoires d'inscription des classes aisées et des zones métropolitaines, est testée en mobilisant une source originale ${ }^{1}$. Il s'agit de l'identification des programmes immobiliers fermés en cours de commercialisation affichés sur les sites Web des promoteurs. Ce recensement a été réalisé en 2007, grâce à l'analyse des descriptifs des programmes. Il actualise un recensement précédent datant de 2002 (Madoré, 2003) et a constitué la première phase

\footnotetext{
1. Pour de plus amples précisions sur les méthodes de recensement des enclaves résidentielles fermées en France, le lecteur pourra se reporter à la référence suivante: Billard et Madoré, 2010, «Une géographie de la fermeture résidentielle en France. Quelle(s) méthode(s) de recensement pour quelle représentation du phénomène? ", Annales de Géographie, $n^{\circ} 675$.
} 
d'un programme de recherche financé par l'Inhes (Institut national des hautes études de sécurité) (Madoré, 2008²). 434 sur 3255 peuvent être considérés comme clôturés et fermés avec contrôle des accès, soit $13 \%$ du total, car ils contiennent l'un des mots clés suivants : "Ensemble clos, clôturé, fermé, protégé, sécurisé ou séparé (par mur, muret, grille, portail automatique, digicode, vidéophone, accès réservé) »; "Résidence située dans un parc clos »; « Présence d'un dispositif de fermeture (par grille, mur, portail) de l'ensemble résidentiel dans sa globalité ». Toutefois, ce recensement reposant sur un mode déclaratif, une sous-estimation de la diffusion réelle du phénomène est certaine car en toute rigueur, seuls les programmes dont le descriptif affichait explicitement et clairement l'existence de la clôture ont été pris en compte.

Les résultats confirment pleinement l'hypothèse d'une diffusion ubiquiste des enclaves résidentielles fermées en France (Billard et Madoré, 2010). Ainsi, $38 \%$ des promoteurs affichent sur leur site Internet au moins une opération immobilière fermée, les sièges sociaux de ces 123 promoteurs (sur un total de 322) étant répartis pratiquement sur toute la France (métropolitaine et Outre Mer).
Par ailleurs, à l'échelle des aires ou unités urbaines françaises, des programmes fermés avec contrôle des accès sont identifiés dans 127 aires ou unités urbaines $^{3}$. Tout l'hexagone est couvert, la densité de points diminuant seulement dans quelques secteurs géographiques de plus faible densité de peuplement, comme le Massif Central ou la ChampagneArdenne (figure 1). Néanmoins, au-delà du caractère ubiquiste du phénomène, les villes méridionales principalement, et secondairement celles de l'est apparaissent bien comme les plus concernées par la diffusion des programmes résidentiels fermés avec contrôle des accès. Quatre aires urbaines du sud de la France (la conurbation azuréenne, Marseille Aix-en-Provence, Montpellier et Toulouse) et trois de la moitié orientale (Dijon, Lyon et Strasbourg) sont ainsi classées dans les dix premières aires urbaines françaises pour le nombre de programmes fermés (entre 8 et 36 en 2007) ou pour leur proportion dans le total de la production locale (entre $15 \%$ et $50 \%$ ).

Autre résultat majeur, cette géographie de la fermeture résidentielle en France n'est aucunement l'apanage des grandes villes. Tous les échelons urbains sont concernés, des plus petites villes à la
Figure 1: Commercialisation de programmes résidentiels fermés par aire ou unité urbaine (2007)

Marketing of gated communities by urban area or unity in France (2007)

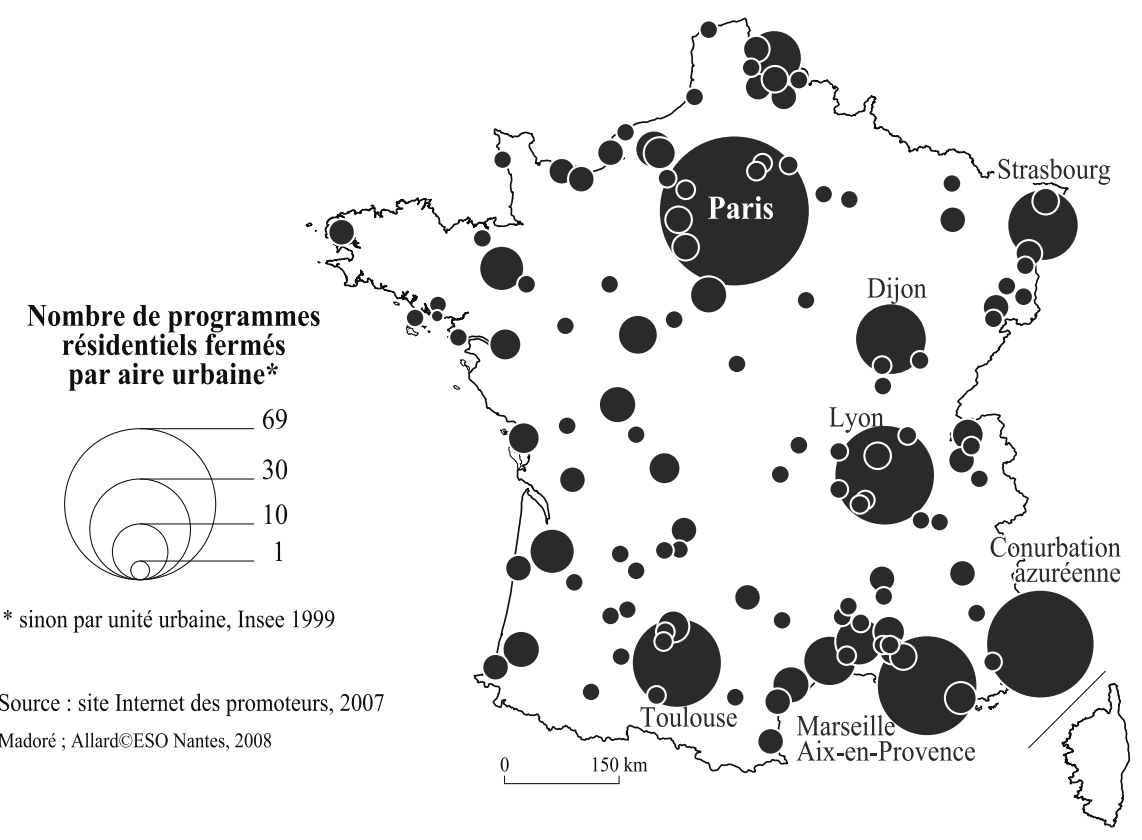

2. Six chercheurs ont travaillé entre 2006 et 2008 sur ce programme intitulé Typologie et représentations des ensembles résidentiels fermés ou sécurisés en France: G. Billard, J. Chevalier, F. Madoré, F. Raulin, A. Taburet, F. Vuaillat.
3. Dans nos publications antérieures, le chiffre de 125 avait été arrêté, car nous avions alors regroupé en une seule entité dénommée "Côte Normande » les petites villes de cette zone géographique possédant des programmes fermés. 
région parisienne. Ainsi, des programmes ont été identifiés dans vingt-deux unités ou aires urbaines de moins de 10000 habitants en 2007, douze ayant même moins de 5000 habitants (tableau 1). Par ailleurs, la localisation de ces petites villes ne révèle aucun effet de concentration géographique bien au contraire, puisqu'elles appartiennent à dix régions métropolitaines plus une située Outre Mer et à dix-huit départements. Si les régions méridionales sont représentées, celles situées plus au nord, à l'image de la Bretagne, de la Basse-Normandie, du Centre ou encore de la Picardie ne sont pas absentes. Certes, dans ces petites villes où l'activité de promotion immobilière est très limitée, un seul programme a été identifié par entité, à l'exception d'une unité urbaine où deux opérations ont été recensées. De fait, elles ne regroupent que $5 \%$ des 434 programmes fermés identifiés en France, mais leur apparition dans ce recensement ainsi que leur répartition sur l'ensemble du territoire français sont très significatifs de leur banalisation géographique.

Cette très large diffusion, bien au-delà des seules métropoles, de nouveaux territoires de l'habiter construits sur le mode de la fermeture et du contrôle des accès est symptomatique d'une recomposition de l'offre immobilière. La réalisation entre 2002 et 2008 de seize entretiens auprès de sociétés de promotion immobilière dans quatre villes différentes (six promoteurs franciliens comptant parmi les plus importants en France, six toulousains, deux martiniquais et un nantais ${ }^{4}$ ) permet ainsi de mieux comprendre comment ces acteurs, qui jouent un rôle clé dans la fabrique du cadre bâti en milieu urbain, ont pris le virage de la fermeture résidentielle au cours de la seconde moitié des années 1990 généralement.

La plupart de ces promoteurs déclarent clôturer systématiquement, ou peu s'en faut, leurs programmes immobiliers et instaurer par la même occasion un système de contrôle des accès. Ils affirment par ailleurs clôturer quel soit le lieu où se situe l'ensemble immobilier en construction, ce qui contribue à la diffusion ubiquiste des programmes fermés avec contrôle des accès. Les plus actifs et les plus précoces dans ce domaine ont été et sont toujours incontestablement les promoteurs dont le siège social est localisé dans le sud de la France, d'où probablement une plus grande visibilité des

\begin{tabular}{|c|cc|cc|}
\hline Taille de l'aire urbaine (AU) ou de l'unité & \multicolumn{2}{|c|}{ Nombre de programmes } & \multicolumn{2}{c|}{ Nombre d'AU ou d'UU } \\
\cline { 2 - 5 } urbaine (UU) & effectif & en \% & effectif & en \% \\
\hline & 13 & 3 & 12 & 9 \\
UU 5 000 habitants ou moins & 10 & 2 & 10 & 8 \\
UU ou AU 5 000 à 9999 habitants & 11 & 3 & 8 & 6 \\
AU 10 000 à 19 999 habitants & 22 & 5 & 19 & 15 \\
AU 20 000 à 49 999 habitants & 36 & 8 & 21 & 17 \\
AU 50 000 à 99 999 habitants & 29 & 7 & 19 & 15 \\
AU 100000 à 199999 habitants & 65 & 15 & 23 & 18 \\
AU 200 000 à 499 999 habitants & 179 & 41 & 14 & 11 \\
AU 500 000 habitants et + & 69 & 16 & 1 & 1 \\
AU de Paris & & & & $\mathbf{1 0 0}$ \\
\hline
\end{tabular}

Source : site Internet des promoteurs, 2007

Tableau 1 : Programmes résidentiels fermés commercialisés en France selon la taille de l'aire ou de l'unité urbaine (2007) (Source : site Internet des promoteurs, 2007)

Gated communities marketed in France according to the size of the urban area or unity (2007)

\footnotetext{
4. Au sein de ces groupes de promotion immobilière, nos interlocuteurs ont été, généralement, le responsable des programmes ou le directeur technique, voire dans les plus petites sociétés, le directeur lui-même. Un promoteur a été interrogé à deux reprises, en 2002 et en 2007, ce qui nous a confirmé l'invariabilité du discours à cinq ans d'intervalle, d'où au total quinze promoteurs interrogés pour seize entretiens réalisés.
} 
ensembles fermés dans les villes méridionales, mais par mimétisme, bien d'autres promoteurs ont intégré cette prestation dans leur production, favorisant ainsi sa banalisation, en particulier géographique.

\section{Recomposition de l'offre immobilière et ordre sécuritaire}

L'existence de ces nouveaux territoires de l'habiter construits sur le registre de la fermeture résulte donc assez largement d'une recomposition de l'offre immobilière, marquée par la promotion de nouveaux services résidentiels à l'échelle du complexe d'habitat. Ces services sont axés d'abord et avant tout sur la sécurisation et le contrôle des accès, mais aussi, du moins pour un certain nombre de complexes, sur la production d'équipements (piscine, courts de tennis, club-house principalement) autorisant une pratique hédoniste et renforçant la légitimité d'un contrôle des accès, dans le but de limiter l'accessibilité aux seuls résidants.

Il s'ensuit alors une banalisation de la rhétorique sécuritaire dans la rédaction des descriptifs des programmes immobiliers neufs en cours de commercialisation, qu'ils soient clôturés ou non d'ailleurs. Cette référence à la sécurité est devenue une sorte de figure imposée, comme l'est également celle du développement durable. Fort logiquement, en lien avec cette banalisation du discours sécuritaire, les promoteurs interrogés déclarent à l'unanimité communiquer vis-à-vis de la clientèle autour de la clôture et des éléments de sécurisation de leurs programmes. Cette mise en évidence d'un argumentaire sécuritaire dans la promotion commerciale d'une opération immobilière s'explique par une conviction partagée par tous les promoteurs : la sécurisation d'un complexe d'habitat, illustrée par sa fermeture à l'aide d'une clôture et d'un contrôle des accès, est devenue une prestation valorisant l'ensemble résidentiel.

Cette réorientation de l'offre immobilière est en phase avec la montée en puissance d'un ordre sécuritaire en France, illustré par la multiplication à partir des années 1970 des discours sécuritaires produits principalement par les acteurs politiques (Robert, 1999; Wieviorka, 1999; Mucchielli, 2001 ; Bonelli, 2008; Rigouste, 2009) : elle en constitue même l'un des symptômes. Les promoteurs estiment en effet qu'il n'est plus guère possible désormais de construire autrement, car cela ne « serait pas dans l'air du temps ». Autrement dit, l'époque étant marquée du sceau de l'insécurité, il devient impératif de promouvoir un habitat sécuritaire, donc fermé avec contrôle des accès. De fait, tous les acteurs qui œuvrent à la production ou à la gestion des espaces résidentiels ont intégré cet impératif sécuritaire. Nombre de syndicats de copropriétaires ou de bailleurs sociaux ont ainsi adopté cette posture, comme l'illustrent à la fois la multiplication des dispositifs mutualisés de contrôle des accès à l'entrée de copropriétés qui n'en possédaient pas jusque dans les années 2000 et le déploiement par les bailleurs sociaux des logiques de résidentialisation, qui aboutissent dans l'un comme dans l'autre cas à la constitution d'enclaves fermées avec contrôle des accès. Cette forte imprégnation des logiques sécuritaires dans l'habitat en France constitue un témoin parmi d'autres de l'inscription durable dans la société française d'un d'ordre sécuritaire adossé à la représentation d'une société qui serait travaillée par de multiples formes d'insécurité, réelles, perçues ou fantasmées.

\section{ChOIX DE DEUX PETITES VILLES DE L'OUEST ATLANTIQUE FRANÇAIS : Biscarrosse et SAINT-Martin- DE-RÉ}

\section{Deux espaces récréo-touristiques}

Qu'est-ce qui se cache derrière cette figure de l'enclave résidentielle fermée? En fait, la réalité est multiforme. Elle ne peut se résumer à une figure générique qui, à elle seule, contiendrait toute la diversité des complexes d'habitat clos. D'où l'intérêt d'observer attentivement ce phénomène en privilégiant l'approche monographique. La finalité étant bien de révéler la diversité typologique des enclaves résidentielles fermées, cet objectif ne peut être atteint que par l'étude de cas de petites villes françaises où le phénomène est bien représenté. Deux d'entre elles, situées dans l'ouest Atlantique français, ont ainsi fait l'objet d'une investigation approfondie, Biscarrosse dans les Landes et SaintMartin-de-Ré en Charente-Maritime.

Ces deux petites villes font parties des onze terrains d'étude choisis en France afin d'y révéler la diversité des enclaves résidentielles fermées. Outre 
ces deux espaces récréo-touristiques, l'échantillon contient également la ville nouvelle de Marne-laVallée en région parisienne, sept aires urbaines de province (Le Mans, Lyon, Nantes, Nice, Rouen, Toulouse et Tours) et un territoire d'Outre mer (la Martinique). Cette typologie, qui constitue la deuxième phase du programme de recherche financé par l'Inhes (cf. supra), est construite à partir d'un échantillon constitué de 200 ensembles immobiliers fermés et d'une grille permettant de renseigner trois séries de variables : des variables morphologiques, d'autres permettant d'apprécier le standing et le style de vie, enfin des variables replaçant le complexe immobilier dans son environnement. Il va de soi, bien évidemment, que cette grille est inégalement remplie d'un ensemble immobilier à l'autre, selon la quantité et la qualité des informations obtenues. Celles-ci ont été recueillies par un minutieux travail de terrain, combinant observations de visu et quête de renseignements auprès de résidants ou de toute personne ressource, comme les gardiens.

Biscarrosse dans les Landes et Saint-Martin-de-Ré possèdent à la fois des traits communs, notamment leur appartenance à un espace récréo-touristique marqué par la forte présence dans l'économie locale du tourisme balnéaire, mais aussi des dissemblances. Il convient dans un premier temps de donner quelques éléments de contexte pour mieux apprécier l'intérêt de ces deux études de cas.

Biscarrosse est une unité urbaine de type ville isolée comprenant 12355 habitants au recensement de 2008. Cette vaste commune à l'échelle du territoire français $\left(160 \mathrm{~km}^{2}\right)$ est constituée de trois noyaux. Biscarrosse-Ville, centre administratif et commercial de la commune situé à une dizaine de kilomètres de l'océan Atlantique, regroupe l'essentiel de la population communale. Les deux autres noyaux, Biscarrosse-Plage au pied du cordon dunaire et Biscarrosse-Lac en bordure du lac Nord à sept kilomètres de Biscarrosse-Ville, ont une vocation balnéaire et touristique très affirmée et comptent relativement peu de résidants permanents. Par ailleurs, cette petite ville bénéficie de la présence importante de deux bases militaires à proximité, avec le Centre d'Essais des Landes (CELM) et la base aérienne de Cazaux. Biscarrosse connaît un rythme d'accroissement démographique très important depuis 1999 : $+3,2 \%$ en moyenne annuelle entre 1999 et 2008 . Cela représente un gain de 3065 habitants en neuf ans. Cette évolution s'explique à $95 \%$ par la vigueur du solde migratoire, les migrations à longue distance contribuant significativement à cette attractivité résidentielle. En effet, 18,5 \% de la population de cinq ans ou plus recensée en 2008 dans cette unité urbaine résidaient cinq ans auparavant dans une autre région de France métropolitaine, soit une proportion trois fois supérieure à celle observée dans l'hexagone $(6,2 \%)$. Par ailleurs, parmi ces individus ayant effectué une migration interrégionale, quasiment un quart $(23,7 \%)$ est à la retraite, ce qui représente plus du double de la proportion observée pour l'ensemble de la France métropolitaine $(11,3 \%)$. Ainsi, la surreprésentation des retraités parmi les migrants qui se sont installés à Biscarrosse dans la première moitié des années 2000 et en provenance d'une autre région que l'Aquitaine renforce une autre caractéristique majeure de la population de cette petite ville, à savoir sa relative vieillesse : $28,1 \%$ de la population est âgée de 60 ans ou plus en 2008 , soit six points de plus $(22,1 \%)$ qu'en France métropolitaine.

Saint-Martin-de-Ré forme avec La Flotte-en-Ré une unité urbaine multicommunale de 5531 habitants en 2008. Seule la commune de Saint-Martin a été étudiée : elle renferme 2585 habitants sur un territoire exigu $\left(4,7 \mathrm{~km}^{2}\right)$. La ville est formée de deux entités bien distinctes, autant par leur histoire que par leurs caractéristiques urbanistiques : d'une part, la ville intra-muros fortifiée par Vauban, constituée d'un tissu urbain dense, tout particulièrement dans la partie ouest d'origine médiévale, alors que la partie orientale, d'époque moderne et de facture néoclassique, est moins dense; d'autre part, une ville extra-muros développée à l'est de la ville fortifiée depuis les années 1970. Principal foyer de peuplement urbain de l'Île de Ré avec la commune voisine de La Flotte, Saint-Martin-de-Ré représente un des points d'ancrage de l'espace récréo-touristique de l'île, dans lequel se marie tourisme balnéaire et patrimonial, comme l'illustre l'inscription de la ville sur la liste du patrimoine mondial de l'Unesco en 2008. Contrairement à Biscarrosse, Saint-Martinde-Ré enregistre une stabilité de sa population, ce qui s'explique largement par la conjonction de deux phénomènes : la saturation d'un territoire communal exigu (550 hab $/ \mathrm{km}^{2}$ en 2008, sachant que le parc de logements est composé essentiellement de maisons); l'amenuisement du parc de résidences 
principales au profit des résidences secondaires, ces dernières représentant désormais $58 \%$ du total des logements. C'est grâce aux apports migratoires que Saint-Martin stabilise sa population, ceux-ci compensant presque en totalité le fort déficit du solde naturel, résultat d'une vieillesse accentuée de la population (28,8\% de 60 ans et plus en 2008). Les migrations à longue distance contribuent à cette attractivité résidentielle, mais selon une intensité bien moindre qu'à Biscarrosse : 10,3\% de la population de cinq ans ou plus recensée en 2008 résidaient cinq ans auparavant dans une autre région de France métropolitaine, soit un peu plus que le taux observé dans l'hexagone (6,2\%). Par ailleurs, parmi ces individus ayant effectué une migration interrégionale, quasiment un cinquième $(18,8 \%)$ est à la retraite, soit significativement plus que la proportion observée pour l'ensemble de la France métropolitaine $(11,3 \%)$.

\section{Recensement des enclaves résidentielles fermées}

Dans ces deux petites villes de Biscarrosse et Saint-Martin-de-Ré, le recensement des enclaves résidentielles fermées repose sur un balayage de terrain rue par rue. Il a été réalisé au cours des années 2007 et 2008. L'intérêt de cette démarche visant l'exhaustivité est de montrer les degrés variables de fermeture et d'accessibilité (piétons et voitures) aux ensembles résidentiels et, in fine, d'esquisser une typologie des modalités du contrôle des accès.

À Biscarrosse-Ville, le boom immobilier qui a accompagné la croissance démographique a été marqué par une proportion significative de construction de nouveaux complexes d'habitat sous forme d'ensembles résidentiels fermés. Sept, réalisés à partir de 2000, ont été recensés (tableau 2; figure 2). Six d'entre eux sont occupés à titre permanent, ce qui représente un total de 309 résidences principales, soit une proportion non négligeable de la construction neuve des années 2000, avec quasiment le quart des résidences principales achevées depuis 1999 sur l'ensemble de la commune. De fait, nous pouvons estimer désormais que $6 \%$ environ des ménages de la commune de Biscarrosse résident dans un complexe résidentiel clos. Ces résidences fermées proposent soit de l'individuel, soit du collectif, mais rarement les deux à la fois (le Domaine Cap Océane étant la seule exception), et l'habitat y est composé en majorité de maisons individuelles (174 contre 135 appartements). Par ailleurs, un complexe est occupé à titre de résidence secondaire : il s'agit du village aéronautique du lac, comprenant 63 villas.

De son côté, Saint-Martin-de-Ré intra-muros connaît, depuis la décennie 1980, un processus de densification sous la forme d'une urbanisation de clos plus ou moins vastes. Ceux-ci sont entourés d'un mur et connaissent des restrictions d'accès variables, allant du registre symbolique à la fermeture totale et permanente. Deux types de clos peuvent être identifiés : ceux constitués d'une parcelle qui avait un usage de jardin potager, de verger ou de parc et qui font l'objet d'une vente au moment du décès du propriétaire; ceux caractérisés par la disparition ou le déplacement de l'activité pré-existante. Dix opérations résultant de l'urbanisation de clos peuvent être identifiées, pour un total de 414 logements (tableau 2; figure 3). L'habitat collectif, toujours limité à deux étages, l'emporte sur l'individuel, avec respectivement 231 et 183 logements. Contrairement à Biscarrosse où les complexes sont rarement mixtes en termes de type d'habitat, cette mixité est un peu plus présente à Saint-Martinde-Ré, puisqu'elle concerne trois ensembles, tandis que cinq offrent exclusivement de l'habitat individuel, mais avec un nombre limité de maisons, et que deux sont composés uniquement d'appartements. Pour chacun de ces clos, le mode d'occupation dominant, voire exclusif dans certains cas, est la résidence secondaire. Ces 414 logements représentent $18 \%$ du parc de logements de la commune en 2008 (2327), cette dernière comprenant également la ville extra-muros. Cette proportion est supérieure à l'emprise spatiale de ces dix complexes, ce qui est assez logique dans la mesure où ceux-ci procèdent d'une logique de densification du tissu urbain.

\section{DIVERSITÉ DES ENCLAVES RÉSIDENTIELLES FERMÉES}

Les dix-sept enclaves résidentielles fermées identifiées à Biscarrosse et à Saint-Martin-de-Ré font écho, du moins partiellement, à la diversité typologique de ces enclaves en France (Billard et al., 2011). Cette variété suggère que les raisons permettant de rendre compte du développement du 


\begin{tabular}{|c|c|c|c|}
\hline \multirow{2}{*}{ Nom de Trmienble rabidentiel ferme } & \multicolumn{3}{|c|}{ 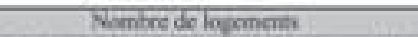 } \\
\hline & Tat & bon inbribel & donim collecti \\
\hline Hliscarmese & 372 & 23 & 135 \\
\hline Dreninarey zesidrege frinsipely & tog & 174 & 135 \\
\hline Les Cols Vens & 10 & & 10 \\
\hline Dumaine Cap Odtane & 36 & 26 & $\omega$ \\
\hline Parc du Moudin & 33 & & 31 \\
\hline La Palonitico: & 32 & & 32 \\
\hline Les Serionithen & 40 & 40 & \\
\hline Vila Vermeil & 108 & 108 & \\
\hline Drminmte tridenser scoondaitse & 63 & 63. & \\
\hline Village atronaticique des lass & $\overline{63}$ & $\overline{65}$ & \\
\hline Salat-Mtartin-de-kt & 4Is & 183 & 231 \\
\hline Desminunte fósidense yosenblaies & & & \\
\hline Clow des Charitains & 23 & 23 & \\
\hline Clos des Geuvemoun & 32 & 20 & 12 \\
\hline Clas de ntertoute & 7 & 7 & \\
\hline Clos Theirat & $\mathrm{x}$ & 8 & \\
\hline Clos Vaiban & 161 & at & 117 \\
\hline Coun de Titleuts & 32 & 32 & \\
\hline Espoce Saire-Clair & 16 & 3 & 5 \\
\hline Residense Baron de Chareal & 35 & & 35 \\
\hline Retiduthe de romeas & 62 & & 62 \\
\hline RGsidenov Re ta Blanste & 18 & 18 & \\
\hline
\end{tabular}

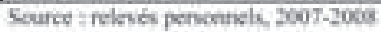

Tableau 2 : Enclaves résidentielles fermées à Biscarrosse et Saint-Martin-de-Ré (2007-2008) Source : relevés personnels, 2007-2008 2008) phénomène sont plurielles. Autrement dit, c'est un faisceau d'éléments qui doit être convoqué, car il n'y a pas une mais plusieurs raisons à cette multiplication d'habitat clos. À chacun des trois types identifiés correspond en fait une raison dominante et d'autres plus secondaires.

\section{Les résidences locatives}

Le premier type d'enclave identifié est composé de cinq résidences locatives, toutes basées à Biscarrosse (tableau 3). Les logiques d'offre sont surdéterminantes pour rendre compte de leur présence dans cette petite ville. En effet, ces résidences ont proliféré partout sur le territoire français depuis les années 1990, en lien avec la succession de mesures de défiscalisation ayant permis à des sociétés à la fois immobilières et financières d'investir le champ de l'immobilier locatif en proposant des produits spécifiques aux investisseurs. Pour les entreprises dominant ce marché, dont l'activité repose sur la trilogie promotion, commercialisation et gestion de biens immobiliers, la fermeture est devenue une prestation permettant de rassurer principalement les investisseurs, à qui l'on vend l'argument d'un moindre risque de dégradation des parties communes extérieures. Ce modèle doit par ailleurs être reproductible dans tous les contextes géographiques, d'autant plus que sa promotion est assurée par un réseau de conseillers vendeurs dont le discours ne peut être que formaté, car destiné à une clientèle d'investisseurs motivés avant tout par la défiscalisation et dispersés sur tout le territoire national. Trois de ces résidences locatives appartiennent d'ailleurs à deux des groupes leaders sur ce marché de l'immobilier défiscalisé et basé à Toulouse, à savoir Akerys (La Palombière) et Omnium Finance (Domaine Cap Océane et Villa Vermeil).

Ces cinq résidences locatives ne sont toutefois pas toutes identiques. Elles diffèrent en particulier par la population qu'elles accueillent et leurs aménités. La plus originale est sans conteste Villa Vermeil. Elle présente la particularité d'avoir été construite à l'origine exclusivement pour les seniors mais, en raison de la vacance d'une partie des logements, a été ouverte ensuite à d'autres ménages. Aujourd'hui, les seniors ne représentent que la moitié du peuplement, occupant une cinquantaine de maisons sur les 108 du complexe. Cette mixité intergénérationnelle imposée alors qu'elle était étrangère à la conception du projet n'est pas toujours bien perçue par les seniors loin s'en faut, certains la vivant comme une trahison et en décalage avec ce qu'ils étaient venus chercher en s'installant ici (Madoré et Vuaillat, 2010). En termes d'aménités, trois de ces résidences locatives en sont dépourvues, en raison notamment de leur taille limitée (moins de 40 logements), alors que les deux autres offrent aux résidants les agréments d'une piscine. Toutefois, héritage du projet initial fondé sur la création d'un village seniors, la piscine de Villa Vermeil est toujours réservée aux seuls seniors qui adhèrent au club Villa Vermeil, cette adhésion étant à la fois discriminée par l'âge et facultative, mais tout en étant payante (125€ mensuel par senior).

\section{Les villages seniors}

Le deuxième type d'enclave résidentielle fermée identifié est un village seniors, en l'occurrence les Senioriales de Biscarrosse. Cette présence montre à l'évidence qu'une nouvelle offre d'habitat perma- 
nent destinée spécifiquement aux seniors valides se développe en France. Si l'apparition de cet entre soi générationnel est assez récent dans l'hexagone (Bésingrand, 2007), il est en revanche bien plus ancien dans d'autres contextes géoculturels, en particulier aux États-Unis, où dès les années 1950 des retirement communities ou active adults communities sont apparus et ont proliféré, en particulier dans les États de la Sun Belt mais pas exclusivement (Stroud, 1995; Pihet, 1999, 2003). Or, cette territorialisation par l'âge se décline souvent sur le registre de la fermeture résidentielle et constitue de fait l'une des catégories emblématiques du phénomène des gated communities étatsuniennes et de la construction de l'entre soi (Blakely et Snyder, 1997; Chevalier et Carballo, 2004).

Les Senioriales de Biscarrosse ont été ouvertes en 2003. Ce concept a été lancé en 2001 par l'ex-groupe Ramos, acteur de la promotion immobilière toulousaine depuis 1973 devenu filiale du groupe Pierre \& Vacances en mai 2007. La notoriété désormais bien établie de ce concept mais aussi de quelques autres comme les Vitabelles ou encore Villages d'Or témoigne de la multiplication des villages seniors mis sur le marché ainsi que de leur diversité, ce que confirme une navigation sur le Web. Ces différents concepts ont des caractéristiques proches que nous retrouvons aux Senioriales de Biscarrosse : ensemble de 40 maisons de plain-pied pour seniors indépendants, en accession à la propriété, offrant à la fois des aménités de loisirs (piscine, club-house, animateur, etc.) et surtout (les sites Internet valorisent cette caractéristique) de sécurité, grâce à la triple présence du portail automatique, de la clôture et du gardien-régisseur (figure 4 a.b.c).

\section{Les villages vacances ou de loisirs}

Enfin, le troisième type d'enclave résidentielle fermée est constitué d'une série de villages vacances ou de loisirs. Par cette appellation, nous désignons des ensembles occupés exclusivement ou essentiellement à titre non permanent, donc à des fins touristiques ou de loisirs. Les dix enclaves identifiées à Saint-Martin-de-Ré et une à Biscarrosse appartiennent à ce type. Autrement dit, l'urbanisation des clos dans la première ville a eu pour conséquence avant tout d'élargir l'offre en résidences secondaires intra-muros. Toutefois, des variations dans l'occu-

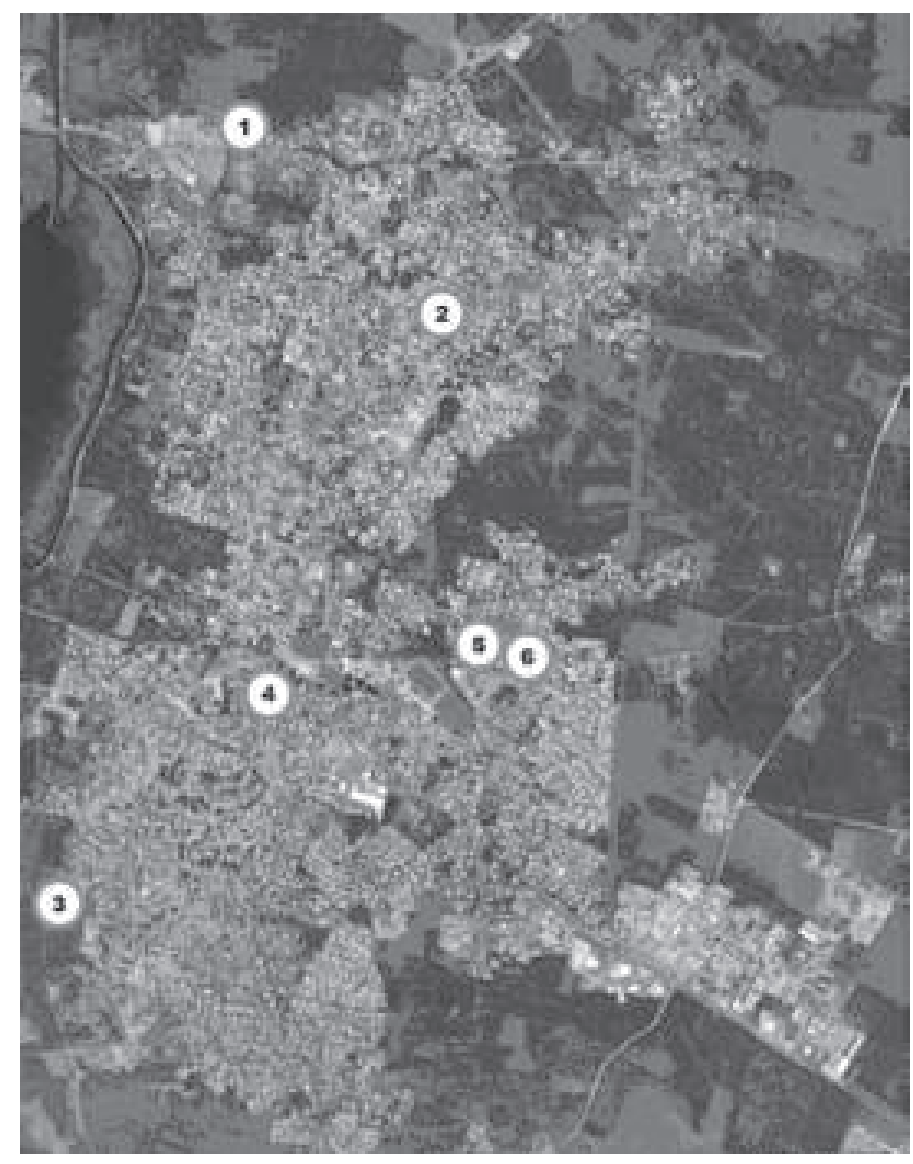

Figure 2 : Enclaves résidentielles fermées à Biscarrosse (2007-2008) 1 : Parc du Moulin, 2 : Domaine Cap Océane, 3 : Les Cols Verts, 4 : La Palombière, 5 : Villa Vermeil, 6 : Les Senioriales.

Gated communities in Biscarrosse (2007-2008)

pation de ces ensembles peuvent être notées, car si certains ne renferment aucune résidence principale, d'autres en contiennent mais en proportion minoritaire. Ces complexes de vacances ou de loisirs sont par ailleurs de taille très variée, le plus petit n'ayant que sept logements (Clos de l'Herbière; figure 5) et le plus grand 161 (Clos Vauban).

Par quel processus cette urbanisation des clos à Saint-Martin-de-Ré s'est-elle accompagnée de leur fermeture? D'une part, ces clos ont été édifiés sur des parcelles déjà encloses par des murs ou des constructions mitoyennes. Il n'a donc pas été nécessaire de construire une enceinte. D'autre part, s'il n'existait pas de restriction totale des accès lorsque ces clos ont été urbanisés, les associations de copropriétaires ont décidé d'ériger au cours de la seconde moitié des années 2000 des obstacles physiques empêchant ou limitant l'accessibilité tnt des voitures que des piétons. Cette volonté de 


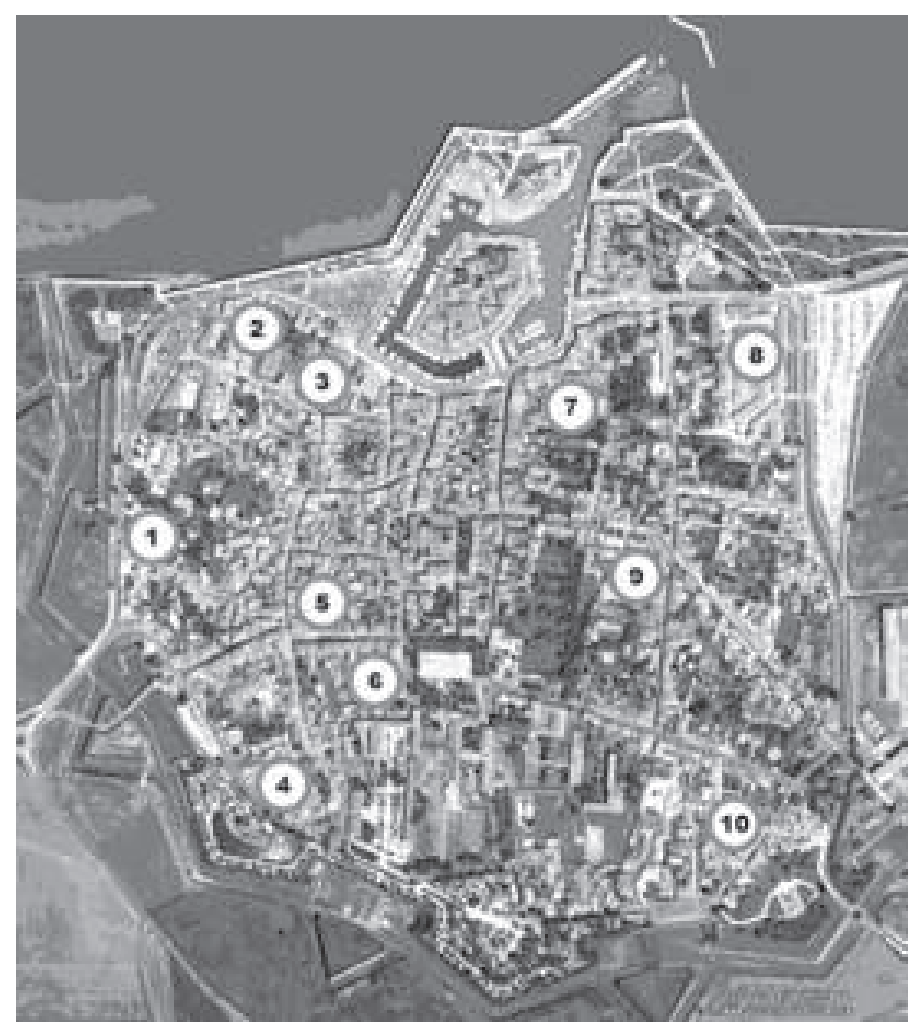

Figure 3 : Enclaves résidentielles fermées à Saint-Martin-de-Ré (2007-2008) 1 : Clos de l'Herbière, 2 : Résidence de l'Orneau, 3 : Résidence Ré la Blanche, 4: Cours des Tilleuls, 5: Espace Saint-Clair, 6: Clos des Gouverneurs, 7 Résidence Baron de Chantal, 8: Clos Vauban, 9: Clos des Charitains, 10 Clos Thoiras.

Gated communities in Saint-Martin-de-Ré (2007-2008)

s'affranchir des inconvénients liés à l'usage (circulation et stationnement) de la voiture en particulier a été observée dans d'autres contextes géographiques, comme le périurbain francilien et lyonnais (Charmes, 2005 ; Loudier-Malgouyres, 2010). À Saint-Martin-de-Ré, ce problème se pose avec acuité principalement lors de la période estivale, où les flux touristiques deviennent très importants. C'est bien cette motivation qui explique, tant au Clos de l'Herbière qu'à la résidence Baron de Chantal par exemple, la pose de portails automatiques hauts de deux mètres environ, qui ont supprimé à la fois l'accessibilité des piétons et aussi toute possibilité pour un non résidant de stationner son véhicule (figure 5). Un autre exemple intéressant à observer est la résidence de l'Orneau, composée d'une rue le long de laquelle s'aligne de part et d'autre une bande continue d'immeubles à $\mathrm{R}+2$ (figure 6 a.b.c). Cet ensemble est fermé en permanence par deux grilles (une à chaque extré-

\begin{tabular}{|c|}
\hline Les rasidenees locathes: \\
\hline $\begin{array}{l}\text { Les Cols Verts (Biscarresse) } \\
\text { Donuine Cap Océane (Biscarnosse) } \\
\text { Pafe du Moulin (Biscarrosse) } \\
\text { La Palombikre (Biscarrosse) } \\
\text { Vilta Vermeil (Biscarrosse) }\end{array}$ \\
\hline Les villages senion \\
\hline Les Senioriales (Bisearrosse) \\
\hline Les réblences de vacances ou de loisin \\
\hline 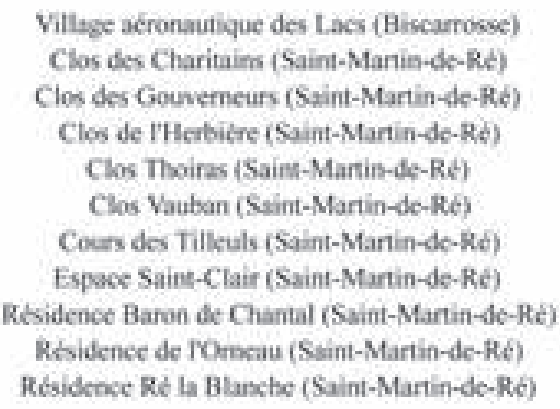 \\
\hline
\end{tabular}

Souree : relevés personnels, $2007-2008$

Tableau 3 : Types d'enclaves immobilières fermées à Biscarrosse et Saint-Martin-de-Ré (2007-2008) (Source : relevés personnels, 20072008) tin-de-Ré (2007-2008)

$$
\text { Type of gated communities in Biscarrosse and Saint-Mar- }
$$

mité), mais celle-ci n'étaient jamais fermées à clé jusqu'en 2007, d'où une accessibilité possible pour les non résidants, à qui cette rue offrait un passage en cœur d'ilot. Or, depuis 2008, l'une des deux grilles est fermée en permanence par l'installation d'un digicode : l'accessibilité est donc toujours possible pour les non résidants qui peuvent entrer par la grille non fermée, mais elle a perdu tout son intérêt, car le digicode supprime de fait la possibilité d'emprunter cette rue pour passer du cours Pasteur à la rue du vieux puits.

Enfin, un cas original de villages vacances ou de loisirs est observé à Biscarrosse, avec le village aéronautique des Lacs (figure 7). Ce concept d'airpark, constitué d'un complexe résidentiel construit autour ou à côté d'un aérodrome, est apparu aux États-Unis après la Seconde Guerre mondiale. En France, le premier village de ce type a été créé en 1996 à Talmont-Saint-Hilaire, au sud de la Vendée, à proximité de la côte Atlantique. Neuf airparks 

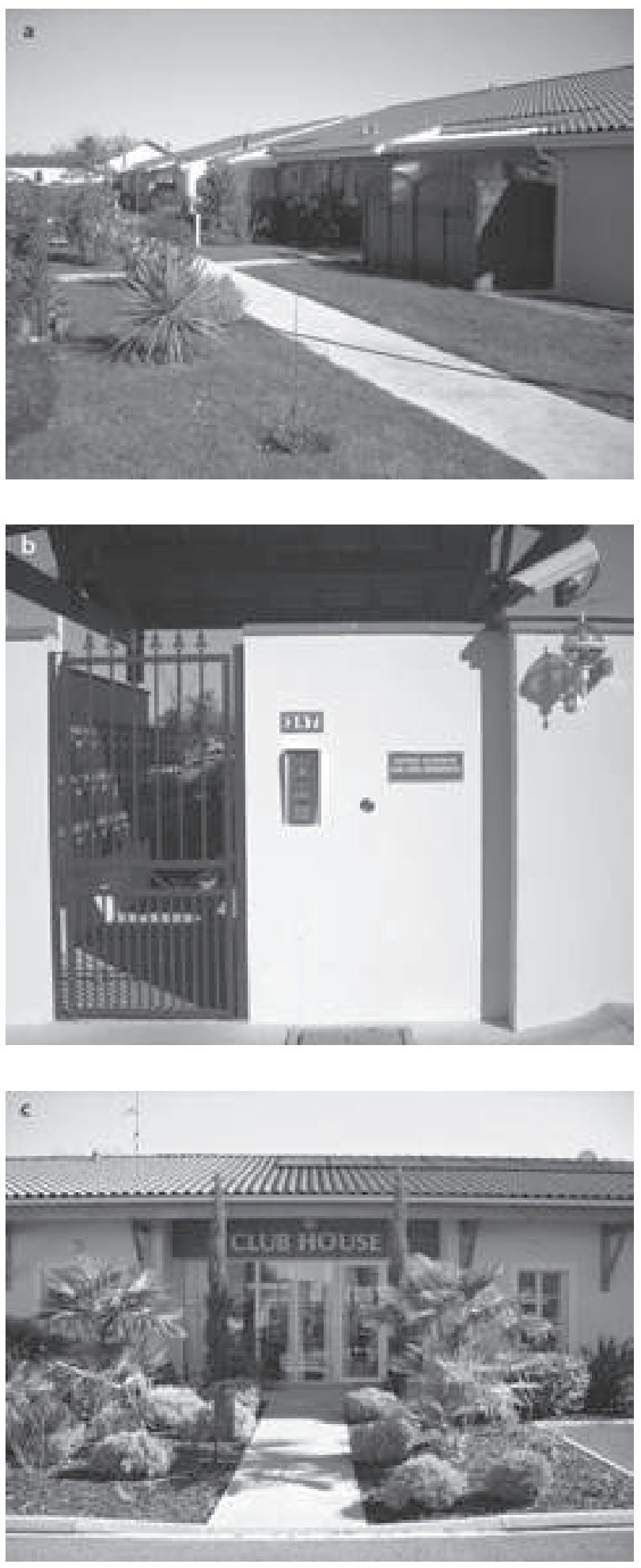

Figure 4 a.b.c : Biscarrosse, Les Senioriales : village seniors Retirement community

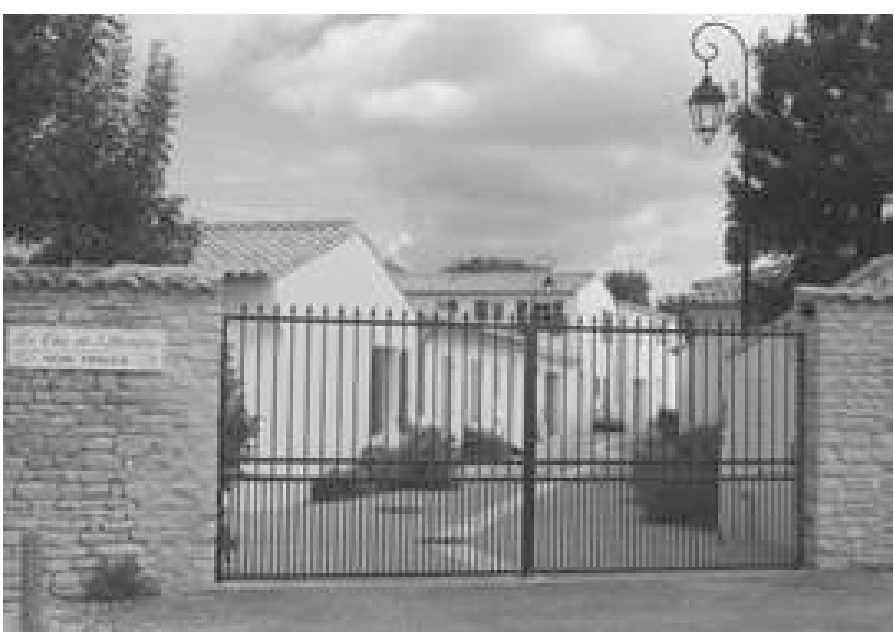

Figure 5 : Saint-Martin-de-Ré, Clos de l'Herbière : installation d'un double portail automatique en 2006

Installation of a double automatic gate in 2006

existent actuellement dans l'hexagone ${ }^{5}$. Celui de Biscarrosse est constitué d'un vaste complexe de 63 grandes maisons avec hangar attenant pour garer l'avion. Les voies privées qui parcourent cet ensemble hermétiquement fermé débouchent directement sur l'aérodrome.

\section{DES MODALITÉS DE CONTRÔLE DES ACCÈS TRÈS VARIABLES}

Si les enclaves résidentielles fermées comportent généralement un contrôle des accès, permettant ainsi de mettre en œuvre l'adage selon lequel «ne rentre plus qui veut », ce contrôle est dans les faits très variable. Il y a en effet de multiples façons de gérer les modalités d'accès des piétons et des voitures. La fermeture peut sembler d'ailleurs plus apparente que réelle. Sur les dix-sept ensembles recensés à Biscarrosse et Saint-Martin-de-Ré, huit sont totalement et en permanence inaccessibles aux non résidants, que ceux-ci soient à pied ou en voiture, alors qu'à l'inverse, dans six complexes, l'accès est possible (tableau 4). Enfin, deux sont inaccessibles pour les voitures mais pas pour les piétons et un présente la configuration inverse. Par ailleurs, à l'image de ce qui observé globalement en France (Billard et al., 2011), la présence d'un gardien est

5. Source : [http://fr.wikipedia.org/wiki/Village_aéronantique]. 

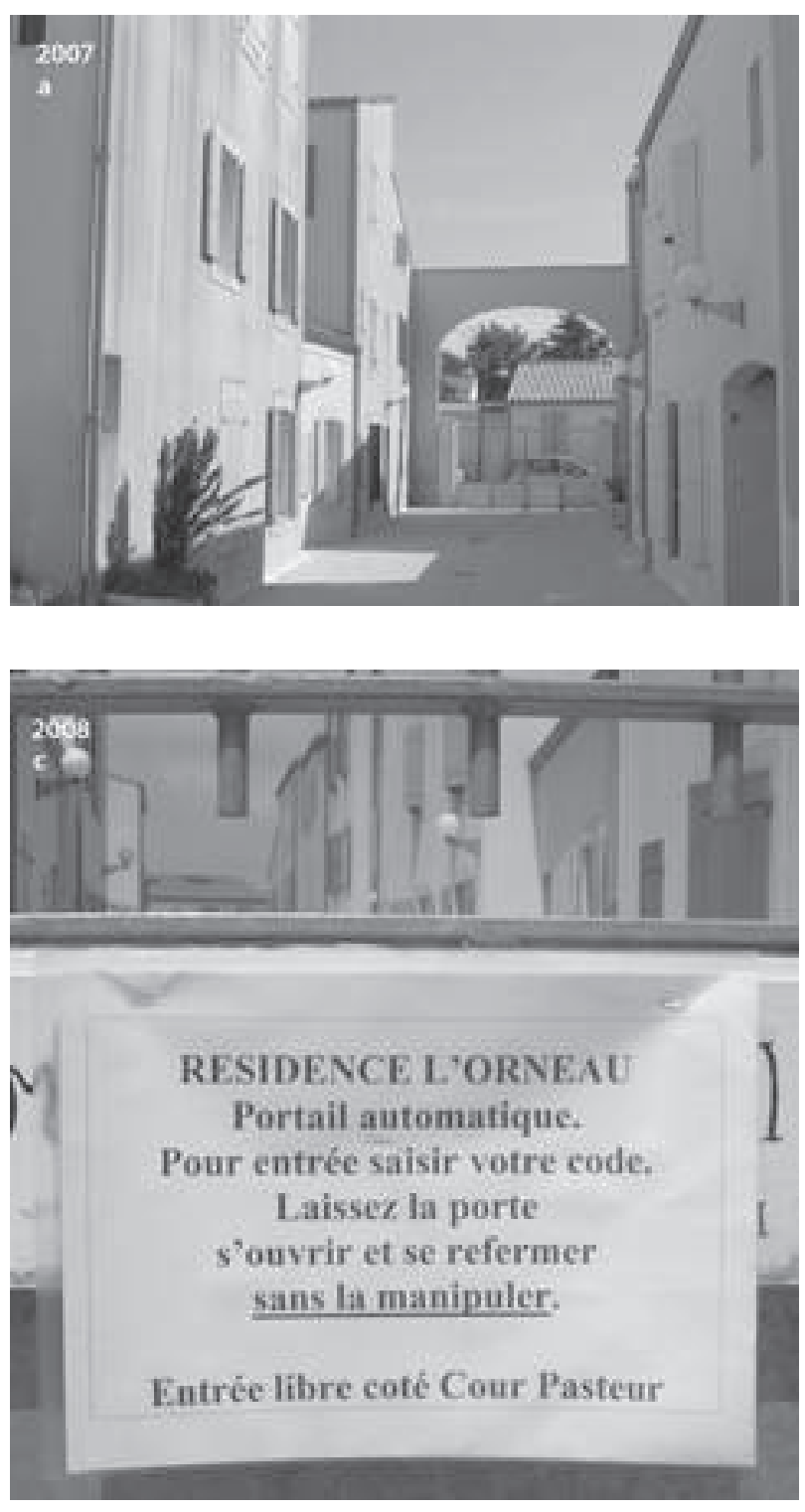

limitée. Seuls trois complexes, situés à Biscarrosse, disposent d'un gardien, mais celui-ci est d'abord et avant tout affecté à l'entretien et à la maintenance, même s'il exerce, au moins de façon informelle, une mission de surveillance des allées et venues. Enfin, aucun des dix-sept complexes recensés ne dispose d'un dispositif de vidéosurveillance.

L'observation fine des modalités d'accès montre que la façon de gérer ces accès pour les non résidants est donc très variable (tableau 3). Ainsi, treize ensembles sur dix-sept offrent un obstacle physique à l'entrée des piétons, généralement un portillon ou une grille. Toutefois, dans quatre cas, l'accès est tout à fait possible, soit parce que ces équipements ne

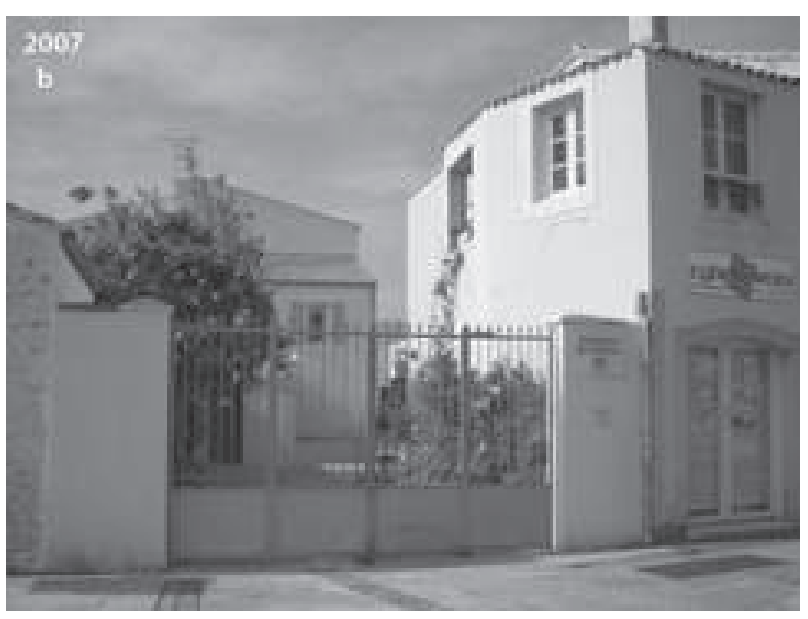

Figure 6 a.b.c : Saint-Martin-de-Ré, résidence de l'Orneau : installation d'un digicode en 2008

Installation of a digicode in 2006

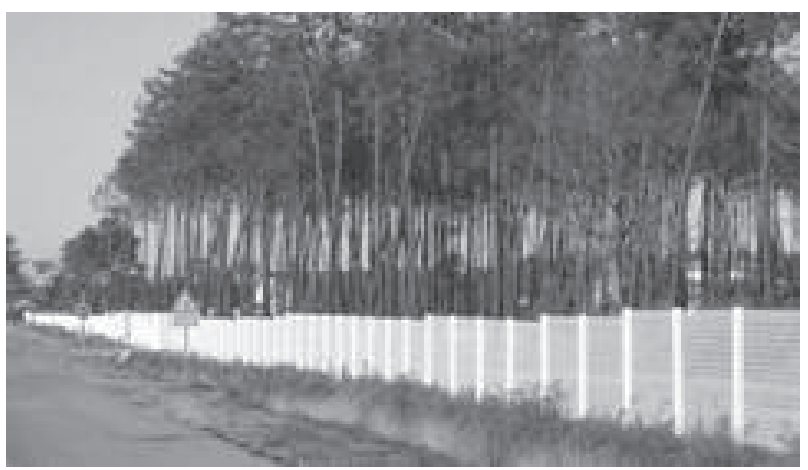

Figure 7 : Biscarrosse, village aéronautique (airpark) des Lacs

sont pas verrouillés, soit parce qu'ils ne fonctionnent pas ou plus. De fait, ces quatre ensembles résidentiels ont toutes les apparences de la fermeture totale et d'une parfaite imperméabilité, mais la réalité est tout autre, car quiconque souhaite rentrer peut le faire. Dans les quatre autres ensembles dépourvus d'un quelconque dispositif technique ou humain de filtrage des allées et venus des piétons (tous situés à Saint-Martin-de-Ré), deux procédés, mis en œuvre la plupart du temps simultanément, signifient très clairement à quiconque qu'il pénètre dans une propriété privée. Le premier passe par la démarcation avec l'espace public, à savoir la rue : celle-ci est fortement marquée à la fois symboliquement et visuel- 


\begin{tabular}{|c|c|}
\hline Modalités d'accés des piétons et des voitures & Effectif \\
\hline $\begin{array}{l}\text { Accés possible pour les piétons et les voitures } \\
\text { Accès possible pour les piétons mais impossible pour les voitures } \\
\text { Accès impossible pour les piètons mais possible pour les voitures } \\
\text { Accès impossible pour les piétons et les voitures }\end{array}$ & $\begin{array}{l}6 \\
2 \\
1 \\
8\end{array}$ \\
\hline TOTAL & 17 \\
\hline Accés piétons & Effectif \\
\hline 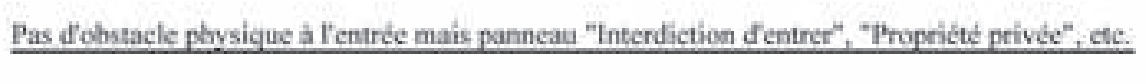 & \\
\hline $\begin{array}{l}\frac{\text { Obstacle physigue à fentrée dont }}{\text { Portillon ou grille non fermés }} \\
\text { Portillon ou grille fermes en parmanence }\end{array}$ & $\frac{13}{4}$ \\
\hline TOTAL & 17. \\
\hline Accés voitures & Effectif \\
\hline Pass dobstacle physigue à fentréce mais obstacle au stationnement avee prósence de chaines & $\underline{2}$ \\
\hline Obstacle physique à Fentróce domt & $\underline{15}$ \\
\hline Portail ou grille non fermés & 2 \\
\hline Chaine à lentréte ou plot amovible & 3 \\
\hline Portail ou grille fermès & 10 \\
\hline TOTAL & 17 \\
\hline
\end{tabular}

Source : relevés personnels, 2007-2008

Tableau 4 : Modalités d'accès des piétons et des voitures dans les ensembles immobiliers fermés à Biscarrosse et Saint-Martin-de-Ré (2007-2008) Equipment to allow access to pedestrians and cars in gated communities in Biscarrosse and Saint-Martin-de-Ré (2007-2008)

lement (par exemple avec une architecture en forme de porte ou de portail), de façon à dissuader toute intrusion humaine. Le second est la présence d'un panneau soulignant l'aspect privatif de la propriété ou rappelant explicitement l'interdiction d'entrer.

L'exemple du Cours des Tilleuls à Saint-Martinde-Ré (figure 8 a.b.c) offre une bonne illustration de la mise en ouvre de ce double procédé. Ce clos ceint de murs et de constructions mitoyennes comporte trois entrées. Si aucune ne comporte d'obstacle physique restreignant formellement l'accès, des dispositifs variés sont activés pour limiter les intrusions. Deux entrées sont accessibles aux véhicules sans aucun obstacle, mais cette possibilité ne présente aucun intérêt, car les places de stationnement sont équipées d'une chaîne (A). Par ailleurs, ces deux entrées sont soulignées par des piliers sur lesquels est apposé le panneau «Propriété privée. Accès réservé » $(B)$, et la différence de revêtement au sol avec l'espace public souligne également l'entrée dans un complexe d'habitat (A). Quant à la troisième entrée, accessible uniquement aux piétons, elle est matérialisée par un porche sous une maison, façon de bien rappeler aussi l'entrée dans un domaine privé, celle-ci étant d'ailleurs rappelée par un panneau mentionnant également « Propriété privée. Accès réservé » $(\mathrm{C})$. 

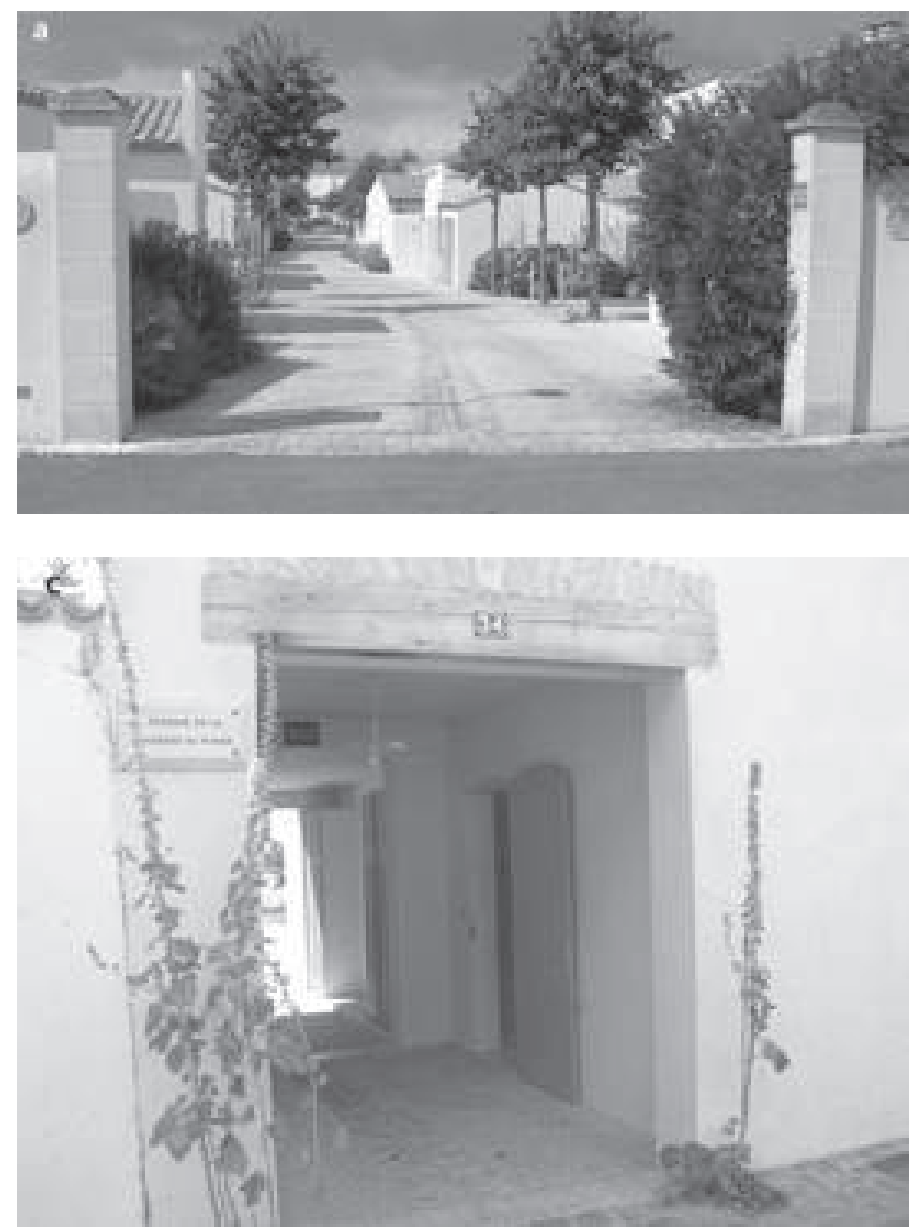

Pour les voitures, quinze des dix-sept ensembles possèdent un obstacle physique empêchant l'entrée et le stationnement des véhicules n'appartenant pas aux résidants (tableau 3). Toutefois, dans deux cas, le portail ou la grille ne sont pas fermés, par défaut de verrouillage ou de réparation d'un portail en panne depuis quelques années, et dans trois cas la restriction d'accès se matérialise par la présence d'une chaîne ou d'un plot amovible à l'entrée. À titre d'exemple, à la résidence La Palombière à Biscarrosse (figure 9), le portail automatique n'a fonctionné que les premiers mois (ouverture de la résidence début des années 2000). Régulièrement, il tombait en panne et un locataire s'ingéniait à le casser. De fait, la résidence est ouverte en permanence, avec un portail ne fonctionnant pas (dernière situation observée : 2008). Enfin, deux complexes n'offrent aucun obstacle physique à l'entrée des voitures mais disposent néanmoins de chaînes au niveau des places de parking, empêchant ainsi physiquement le stationnement des véhicules non autorisés.

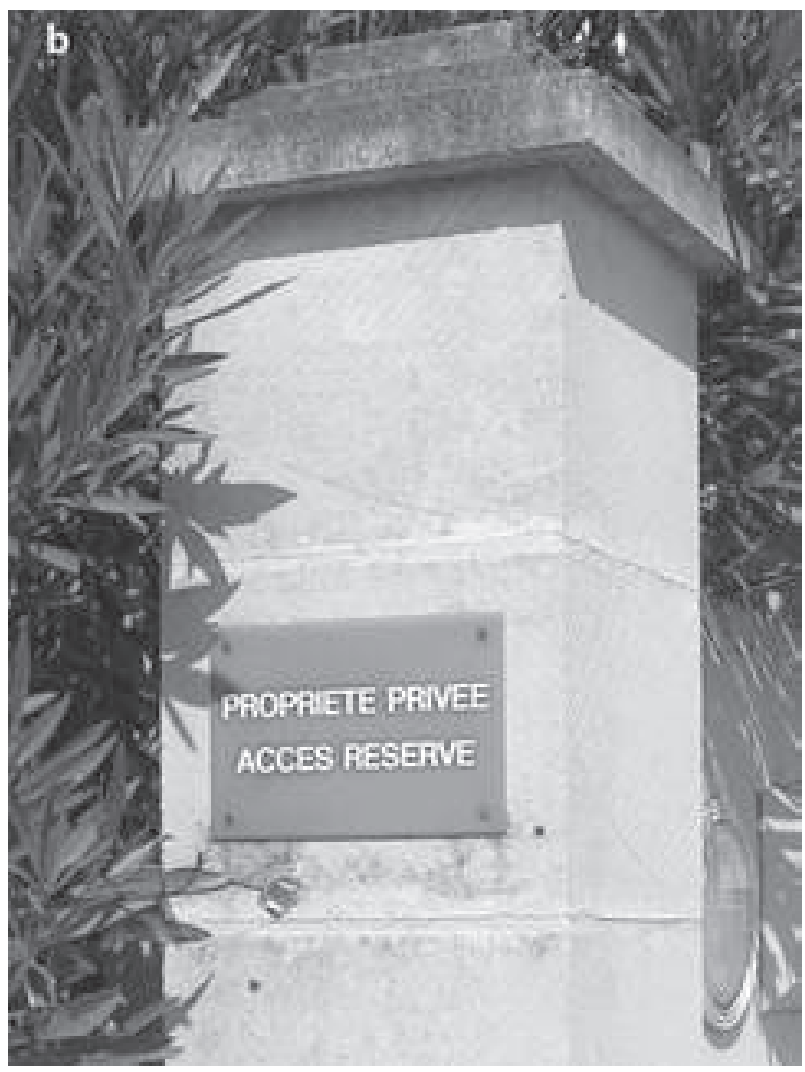

Figure 8 a.b.c : Saint-Martin-de-Ré, Cours des Tilleuls, pas de portail automatique

No automatic gate

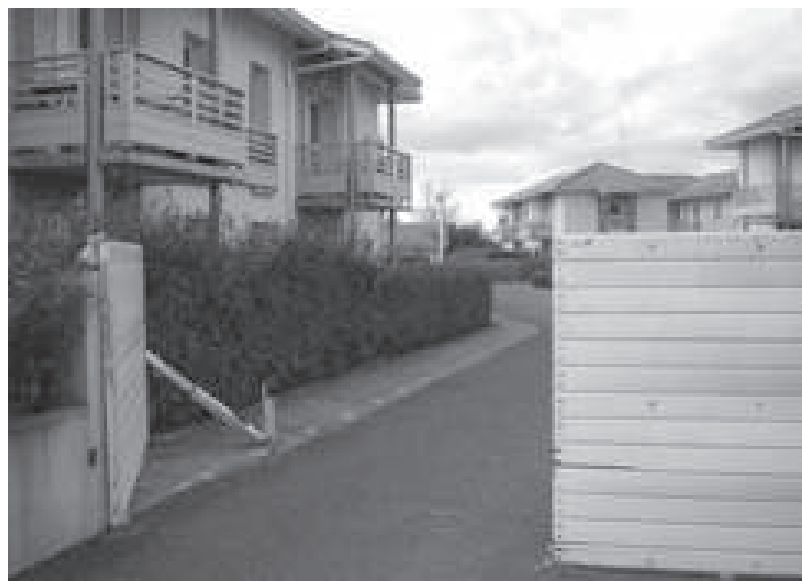

Figure 9 : Biscarrosse, La Palombière : portail automatique en panne depuis des années

Automatic gate out of order for years 
Cette diversité de contrôle des accès n'est pas spécifique aux deux petites villes observées. Elle est également présente dans les neuf autres sites investigués en France (Billard et al., 2011) et a été bien mis en évidence aussi en Île-de-France par LoudierMalgouyres (2010), dont les travaux révèlent que nombre d'ensembles vendus comme fermés ne le sont plus en réalité, soit parce qu'ils ont été ouvert par la suite, soit parce que les habitants se sont lassés des dispositifs de contrôle des accès. Cela accrédite l'idée que si la fermeture et la sécurisation sont considérées par les promoteurs comme un argument vendeur, les résidants en relativisent quant à eux l'intérêt, comme nous l'avons montré en comparant le discours des promoteurs en France avec le regard des habitants (Madoré et Vuaillat, 2009). Enfin, à l'image de ce qui est observé à Saint-Martin-de-Ré, la fermeture peut aussi se décliner en région parisienne sur un registre symbolique, en étant marquée par la présence d'un « seuil d'entrée » qui rappelle le caractère privatif de l'ensemble (LoudierMalgouyres, 2010).

La prolifération d'ensembles résidentiels fermés et/ou sécurisés n'est l'apanage d'aucun contexte géographique en France. Les petites villes sont autant concernées que les métropoles, car les logiques qui président au développement du phénomène résultent avant tout de l'imposition au cœur de la société française d'un ordre sécuritaire qui devient l'une des marques de fabrique de l'émergence d'un «État libéral-autoritaire » (Fœssel, 2010). Les murs symbolisent alors cette tension entre ouverture au monde et nécessité de se protéger d'un environnement perçu comme menaçant. Cet ordre sécuritaire imprime sa marque à la gestion des territoires, quelle que soit leur localisation, amenant l'ensemble des acteurs de l'habitat à succomber à la surenchère sécuritaire : les promoteurs conçoivent des programmes fermés avec contrôle des accès et «vendent de la sécurité » dans leur discours promotionnel; les bailleurs sociaux érigent grilles et codes d'accès en multipliant les opérations de résidentialisation; quant aux assemblées de copropriétaires, elles votent à tour de bras la mise en place de dispositifs de contrôle des accès à l'entrée des copropriétés construites avant la tendance à la généralisation de ces dispositifs au cours des années 1990 et 2000.

Bien évidemment, cette prolifération de murs et de contrôles d'accès n'est pas sans incidence sur les modes d'habiter, car au-delà d'une quête chimérique de sécurité, se profile une recherche de maîtrise de l'environnement résidentiel. Cette quête renvoie à une volonté de contrôler son «chez-soi » et d'assurer sa tranquillité. Elle dépasse donc toute préoccupation sécuritaire au sens strict. Il s'agit plus prosaïquement d'interdire au non résidant, même dénué de toute intention malveillante, de pénétrer dans le domaine résidentiel, qu'il soit piéton et encore plus automobiliste. L'exemple du complexe résidentiel le Parc du Moulin à Biscarrosse en offre une bonne illustration. C'est un ensemble jouxtant le centre commercial de l'avenue Laouadie (route de Biscarrosse-Plage), avec d'un côté le centre Leclerc et de l'autre des moyennes ou grandes surfaces spécialisées. L'entrée de la résidence se fait à partir du parking du centre commercial, devant les magasins spécialisés. Du fait de cette situation (proximité immédiate d'une route passagère et d'une zone commerciale), la fermeture et la pose d'un portail automatique permettent d'assurer une relative tranquillité aux locataires de cette résidence locative comprenant 33 appartements, en évitant en particulier que les aires de stationnement réservées aux résidants ne servent de parking relais au centre commercial.

De fait, cette multiplication des barrières et autres limitations d'accès aux complexes résidentiels modifie les usages de la ville. Certes, l'essor des enclaves résidentielles fermées ne traduit pas une extension de l'espace privé au détriment du domaine public, mais matérialise, par l'imposition d'une limite claire et/ou d'un contrôle des accès, la différence de statut juridique entre ces deux espaces. Ce processus traduit donc, comme l'ont montré divers travaux conduits en particulier sur Toulouse (Golovtchenko et Souchet, 2005; Sabatier, 2005), une extension des espaces de transition, entre le privé intime et le domaine public ouvert à tous. Toutefois, si on privilégie l'accessibilité des lieux, une rétraction des espaces considérés, d'un strict point de vue de leur usage, comme relevant du domaine public, est bien à l'œuvre, car la fermeture induit une accessibilité réduite aux seuls résidants. Le fait de fermer, par exemple, un passage privatif entre deux immeubles d'une même copropriété peut sembler tout à fait légitime au regard de l'application du droit de propriété, mais être perçu, en même temps, comme la suppression d'un itinéraire ayant les apparences et 
les fonctionnalités d'un espace public. C'est bien ce que l'on observe par exemple à Saint-Martin-de-Ré, où la multiplication des restrictions d'accès aux clos modifie la géographie des parcours possibles pour les piétons. Certain d'entre eux ne sont désormais plus envisageables, obligeant alors le passant à modifier et souvent à allonger son itinéraire. La municipalité de Saint-Martin s'efforce de convaincre les assemblées de copropriétaires de laisser au moins un passage pour les piétons, donc de limiter la restriction d'accès aux seules voitures, mais ce souhait n'est pas toujours exhaussé, car le code de l'urbanisme ne peut aucunement intervenir sur l'usage privatif de la parcelle ou des biens communs d'une copropriété, donc empêcher la fermeture des complexes d'habitat (Le Louarn, 2003).

\section{Bibliographie}

BÉSINGRAND D., 2007. La marginalisation des communautés de seniors en France et au Portugal, Bulletin de l'Association des Géographes Français, n³ 3, p. 327-336.

Billard G., Chevalier J., Madoré F., 2005. Ville fermée, ville surveillée. La sécurisation des espaces résidentiels en France et en Amérique du Nord, Rennes, PUR, 230 p.

Billard G., Madoré F., 2010. «Une géographie de la fermeture résidentielle en France. Quelle(s) méthode(s) de recensement pour quelle représentation du phénomène? ", Annales de Géographie, n 675, p. 492-514.

Billard G., Chevalier J., Madoré F., Vuaillat F., 2011. Quartiers sécurisé : un nouveau défi pour la ville?, Paris, Les Carnets de l'info, $205 \mathrm{p}$.

Blakely E.J., Snyder M.G., 1997. Fortress America : Gated Communities in the United States, Cambridge, Washington (DC), Brooking Institution Press/Lincoln Institute of Land Policy, 208 p.

Bonelli L., 2008. La France a peur. Une histoire sociale de l'insécurité, Paris, La Découverte, 420 p.

Caldeira T., 1996. «Un nouveau modèle de ségrégation spatiale : les murs de São Paulo », Revue internationale des sciences sociales, $\mathrm{n}^{\circ} 147$, p. 65-77.

Caldeira T., 2001. City of Walls. Crime, Segregation, and Citizenship in São Paulo, Berkeley, University of California Press, 504 p.

Callen D., Le Goix R., 2007. Fermetures et « entre soi » dans les enclaves résidentielles, dans SAint-Julien T., Le Goix R. (dir.), La métropole parisienne. Centralités, inégalités, proximités, Paris, Belin, p. 211-233.

Capron G., 2006, Quand la ville se ferme. Quartiers résidentiels sécurisés, Paris, Bréal, 288 p.

Charmes É., 2005. La vie périurbaine face à la menace des gated communities, Paris, L'Harmattan, 219 p.
Chevalier J., Carballo C., 2004. Fermetures résidentielles et quête de l'entre-soi, entre Nord et Sud des Amériques, L'Espace géographique, $\mathrm{n}^{\circ}$ 4, p. 325-335.

Donzelot J., 1999, La nouvelle question urbaine, Esprit, $n^{\circ} 11$, p. $87-114$.

Dorier-Apprill É., Audren G., Garniaux J., Stoupy A, Oz R., 2008. Ensembles résidentiels fermés et recompositions urbaines à Marseille, Pouvoirs Locaux, n 78, p. 92-98.

Fessel M., 2010. État de vigilance. Critique de la banalité sécuritaire, Lormont, Le Bord de l'eau, 155 p.

Giroir G., 2002. «Le phénomène des gated communities à Pékin, ou les nouvelles cités interdites », Bulletin de l'Association des Géographes Français, n 4, p. 423-436.

Glasze G., Webster C., Frantz K., 2002. The global spread of gated communities, Environment and Planning, B 29, $\mathrm{n}^{\circ} 3$, p. 315-320.

Glasze G., Webster C., Frantz K. (dir.), 2005. Private Cities. Global and Local Perspectives, Londres, Routledge, 242 p.

Golovtchenko N., Souchet F., 2005. « Des gated communities à la française? Les résidences fermées toulousaines », in Haumont B., Morel A. (dir.), La société des voisins, Paris, Éditions de la Maison des sciences de l'homme, p. 145-167.

Jaillet M.-C., 1999. Peut-on parler de sécession urbaine à propos des villes européennes?, Esprit, n 11, p. 145-167.

Lacarrieu M., Thuillier G., 2004. «Une utopie de l'ordre et de la fermeture : "quartiers privés" et "countries" à Buenos Aires ", L'Espace géographique, n² 2, p. 149-164.

Le Goix R., 2002. « Les gated communities à Los Angeles, place et enjeux d'un produit immobilier pas tout à fait comme les autres ", L'Espace géographique, n 4, p. 328-344.

Le Goix R., Webster C., 2008. Gated Communities, Geography Compass, 2/4, p. 1189-1214.

Le Louarn P., 2003. Les villes fermées ou l'impuissance du droit, Études foncières, $\mathrm{n}^{\circ} 105$, p. 17-23.

Loudier-Malgourres C., 2007. « L'effet de rupture avec l'environnement voisin des ensembles résidentiels enclavés. Une approche morphologie de l'enclavement résidentiel en France ", Les Annales de la Recherche Urbaine, n 102, p. 69-77.

Loudier-Malgourres C., 2010. L'enclavement et la fermeture des ensembles d'habitat individuel. Première approche à partir des aspects morphologiques en Île-de-France, Paris, IAU Îlede-France, $106 \mathrm{p}$.

Madoré F., 2003. « Logiques géographiques et sociales de l'enclosure des espaces résidentiels en France », Belgeo, $\mathrm{n}^{\circ} 1$, p. 99-113.

Madoré F. (dir.), Billard G., Chevalier J., Taburet A., Vuaillat F., Raulin F., 2008. Typologie et représentations des ensembles résidentiels fermés ou sécurisés en France, Inhes, $159 \mathrm{p}$.

Madoré F., Vuaillat F., 2009. « Les logiques sécuritaires et dans le discours des promoteurs et des résidants des ensembles résidentiels fermés : l'exemple de Nantes ", Norois, $\mathrm{n}^{\circ} 212$, p. $9-22$

Madoré F., Vuaillat F., 2010, Une affaire de générations : la construction d'un entre-soi à l'épreuve de la mixité intergénérationnelle. L'exemple de la Villa Vermeil de Biscarrosse, 
EspacesTemps.net, Textuel, 28.06.2010 [http://www.espacestemps.net/document8233.html].

Mucchielli L., 2001. Violences et insécurité. Fantasmes et réalités dans le débat français, Paris, La Découverte, 142 p.

Paquot T. (dir.), 2009. Ghettos de riches. Tour du monde des enclaves résidentielles sécurisées, Paris, Perrin, 296 p.

Pinet C., 1999. Le développement d'une territorialisation produite par l'âge : les « retirement communities » aux ÉtatsUnis, Annales de géographie, n 608, p. 420-435.

Pinet C., 2003. Vieillir aux États-Unis. Une géographie sociale et régionale des personnes âgées, Rennes, PUR, $253 \mathrm{p}$.

Pinçon M., Pinçon-Charlot M., 2007. Les Ghettos du Gotha : Comment la bourgeoisie défend ses espaces, Paris, Seuil, $294 \mathrm{p}$.

Raposo R., 2003. « New Landscapes: gated housing estates in the LisbonMetropolitan Area », Geographica Helvetica, $n^{\circ} 4$, p. 293-301.
Rigouste M., 2009. L'ennemi intérieur. La généalogie coloniale et militaire de l'ordre sécuritaire dans la France contemporaine, Paris, La Découverte, $341 \mathrm{p}$.

Robert P., 1999. Le citoyen, le crime, l'État, Genève, Droz, $311 \mathrm{p}$.

SABAtier B., 2005. «Les résidences fermées à Toulouse : une offre immobilière construisant la "ville privée" », Espaces et sociétés, $\mathrm{n}^{\circ} 123$, p. 131-149.

STROud H., 1995. The promise of paradise: recreational and retirement communities in the United States since 1950, Baltimore, Johns Hopkins University Press, 228 p.

Wieviorka M., 1999. Violence en France, Paris, Seuil, 352 p. 